\title{
Landmine Detection Technology Research in the Netherlands
}

\author{
H.M.A. Schleijpen \\ Programme manager demining research \\ TNO-Physics and Electronics Laboratory \\ P.O. Box 96864, 2509 JG The Hague, The Netherlands \\ schleijpen@fel.tno.nl
}

\begin{abstract}
This paper gives an overview of the activities on research and development in the technology area for landmine detection in the Netherlands. The main players, their projects and the long term and short term project goals are presented. The projects cover the range from military applications to humanitarian demining.

In the "conventional" detection systems area the activities on Metal detection, Ground Penetrating Radar and Thermal Infrared are covered. Signal processing and Sensor fusion are key activities in this area and examples of these activities are shown as well. The focus for these techniques is on vehicle mounted and airborne multi-sensor systems. The activities are supported by more fundamental modelling of the interaction of sensors with the landmines and especially the effects of the environment of the mines on this interaction.

In the area of more future oriented techniques the following techniques are discussed: forward looking Polarised Infrared for moving platforms, Neutron Backscattering techniques and Laser Vibrometry for acoustic detection.
\end{abstract}

Keywords: Landmine detection technology, Sensor Fusion, Metal detection, Ground Penetrating Radar, Polarised Thermal Infrared, Multisensor Systems, Neutron Backscattering and Laser Vibrometry.

\section{INTRODUCTION}

This paper presents the landmine detection technology research in the Netherlands. The main research organisations in this area are: TNO, TUD-IRCTR (Delft University of Technology and International Research Centre for Telecommunications-transmission and Radar) and TUD-IRI (Interfaculty Reactor Institute). The main goal of the research programme at TNO is to contribute to the development of technology for vehicle mounted and airborne multisensor systems. The main emphasis in the programme is on signal processing and sensor fusion of the sensors in a multi-sensor environment. Signal processing should extract features from the signals, which are subsequently used in sensor fusion. For an optimum performance of the sensors understanding of the limitations of the sensors induced by the environment like soil type, soil structure, humidity, temperature, vegetation etc. is necessary. TUD-IRCTR focuses on GPR hardware technology and signal processing, while TUD-IRI concentrates on neutron backscattering techniques. The TNO-programme is mainly sponsored by the Netherlands Ministry of Defence (MoD), while the MoD also sponsors the TUD-IRCTR and TUD-IRI research. Several TNO-projects have been co-funded by the European Commission. The STW-organisation funds the major part of the GPR research at TUD-IRTCR. The Technology Foundation STW is the Dutch funding agency for university research in technical areas.

Although the application of landmine detection technology may differ in a military setting from application in a humanitarian setting, on the technology level itself, the discrimination is less distinct. Apart from this, most of the current operations of the Netherlands Armed Forces are in peace-keeping missions. In this type of operations the application of countermine technology comes much closer to the way the technology is used in humanitarian demining. Feedback from potential users of the technology is essential. Therefore, frequent discussions with both military countermine specialists and humanitarian deminers are part of the project planning. 


\section{DETECTION SENSORS AND SIGNAL PROCESSING}

\subsection{Metal Detection}

As a part of the programme at TNO the metal detector output has been analysed to extract more information from the signals than just the presence of metal. The depth of the metal object can be extracted under certain conditions from the signal variations when the Metal Detector (MD) coils are moving over the object [1]. The signal features caused by the difference in metal type are also analysed and have shown to be much weaker and therefore much more difficult to extract.

\subsection{Ground Penetrating Radar}

The major part of the Ground Penetrating Radar (GPR) research for landmine detection in the Netherlands is carried out at the Delft University of Technology (DUT) and IRCTR (International Research Centre for Telecommunicationstransmission and Radar) in Delft, in co-operation with TNO. IRCTR/DUT have developed and built two GPR hardware concepts: a VIRLAD (Video Impulse Radar for Landmine detection) and an SFCW (Stepped Frequency Continuous Wave) system. On signal processing IRCTR/DUT works together with TNO.

VIRLAD has been initially been developed under sponsorship of the Netherlands MoD. Analysis of the system performance, based on trials at the TNO-FEL test site (Section 5), has shown that the working system bandwidth is considerably larger than that of major commercially available GPR. Due to good matching of transmitting antenna to the impulse generator a very low level of trailing oscillations in a backscattering signal is achieved. Together with short duration of the radiated signal this leads to very fine down-range resolution.

The VIRLAD system was further developed to a Polarimetric Video Impulse Radar [2] within the STW project "Advanced re-locatable multi-sensor system for buried landmine detection". Also the SFCW radar has been developed within this project [3].

The overall aim of the project is to investigate the feasibility of using GPR with integration of multiple microwavesensor technologies to improve the individual landmine detection. The research activities in this project focus on:

a) Multi-sensor signatures of landmines and soils,

b) Development of systems capable of detecting and locating landmines,

c) Subsurface polarimetric UWB SAR technique,

d) Polarimetric UWB Interferometry technique,

e) Location of objects using EM modelling technique,

f) High resolution subsurface imaging technique and

g) Data fusion and object classification technique.

The project distinguishes three parts:

Part 1 comprises the design and realisation of two re-locatable imaging GPR systems. This research concentrates on the development of the ultra-wideband (UWB) impulse radar and the (SFCW) radar.

The stepped frequency radar (SFCW) has been set to work in September 2002. An inherent part of the developed hardware is new antennas. New spiral antennas, developed for SFCW radar, have been measured (figure 1). Several measurements have been made in order to find the optimum geometry for antenna system.

Part 2 comprises the development of accurate electromagnetic modelling methods, high resolution 3-D image reconstruction, robust signal and image processing and experimental verification. Signal processing and imaging methods are evaluated, verified and modified by executing outdoor experimental campaigns. During 2002 the first priority in these activities was given to experimental verifications of the algorithms. To this end a joint measurement campaign took place at the TNO-FEL test lanes. TU Delft has participated in this campaign with VIR with the relocatable scanner, whereas TNO participated with a Metal Detector array, Thermal imagers and Polarised Infrared camera's. During this measurement campaign the impact of the soil water content has been investigated. The test facility contains a number of moisture sensors buried at various depths around land-mines

Part 3 comprises the development of multi-sensor data fusion (MSDF), feature extraction and object classification methods and algorithms. The research on feature extraction and object classification focused on studying the influence of target and soil properties, as well as depth of burial on features extracted from GPR images. Understanding this influence is crucial for designing a classifier that uses these features for mine identification. 


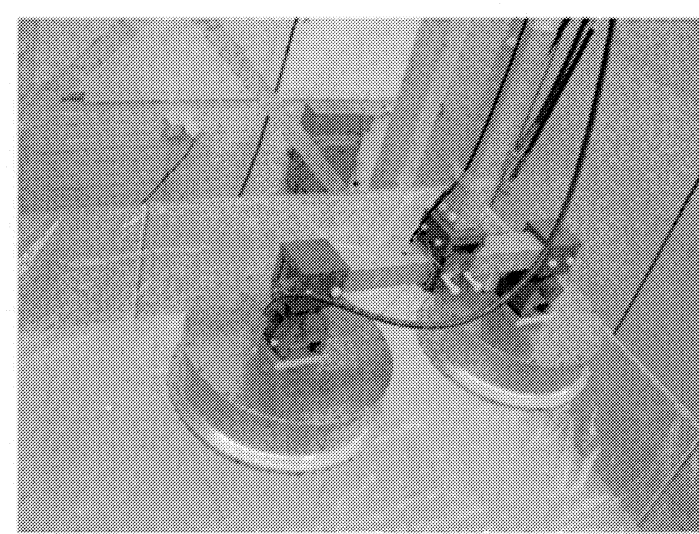

Figure 1. Measurements with the stepped-frequency system CW GPR system in the sand box

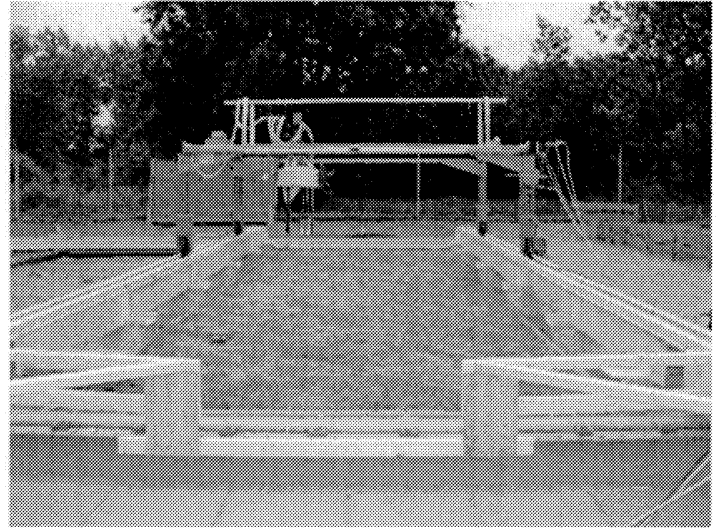

Figure 2. Video impulse GPR system mounted on the relocatable scanner at the test lanes at TNO-FEL.

\subsection{Electro-Optics}

In the wavelength range for Electro-Optical (EO) sensors, detections are obtained for surface laid mines or very shallow buried mines. The most commonly used EO technique for landmine detection is Thermal Infrared (TIR) imaging for detection of surface laid mines.

If a sensor is used in the appropriate part of the electro-optical spectrum, discrimination between landmines and vegetation is possible. Techniques which have been investigated are Multi-spectral imaging [4] and infrared polarisation [5]. These techniques can be used for detection of mines in vegetation and have potential for detection of tripwires. Figure 3 shows the difference in contrasts in several selected spectral bands in the visual part of the spectrum . Figure 4 shows the effect of polarisation for detection of mines partially covered by vegetation.

In order to be able to increase the speed of vehicle mounted systems one needs forward looking sensors. In the same measurement campaign mentioned above under the GPR section, the first measurements with a polarising thermal infrared camera were performed from a moving platform. The reconstructed image of the test lane is shown in figure 5. Also laser vibrometry has potential as a forward looking sensor. Laser pulses are used to heat the top level of the soil and induce a pressure wave of on the soil. The laser vibrometer then probes the soil surface for differences in vibrations in the soil above landmines compared to their environment. The technique has been demonstrated in recent experiments at the TNO test lanes [6]. In these experiments the laser excitation technique was compared to the results of acoustical excitation techniques.
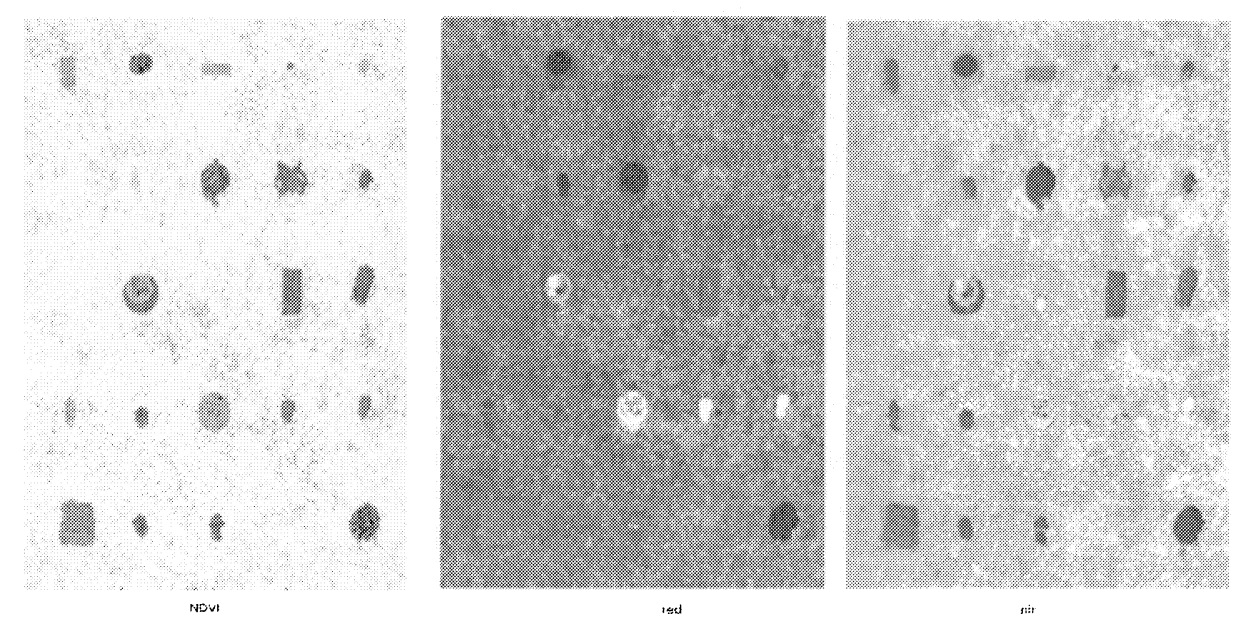

Figure 3: Contrasts of surface laid mines against a grass background in selected bands in the visual to near infrared. 

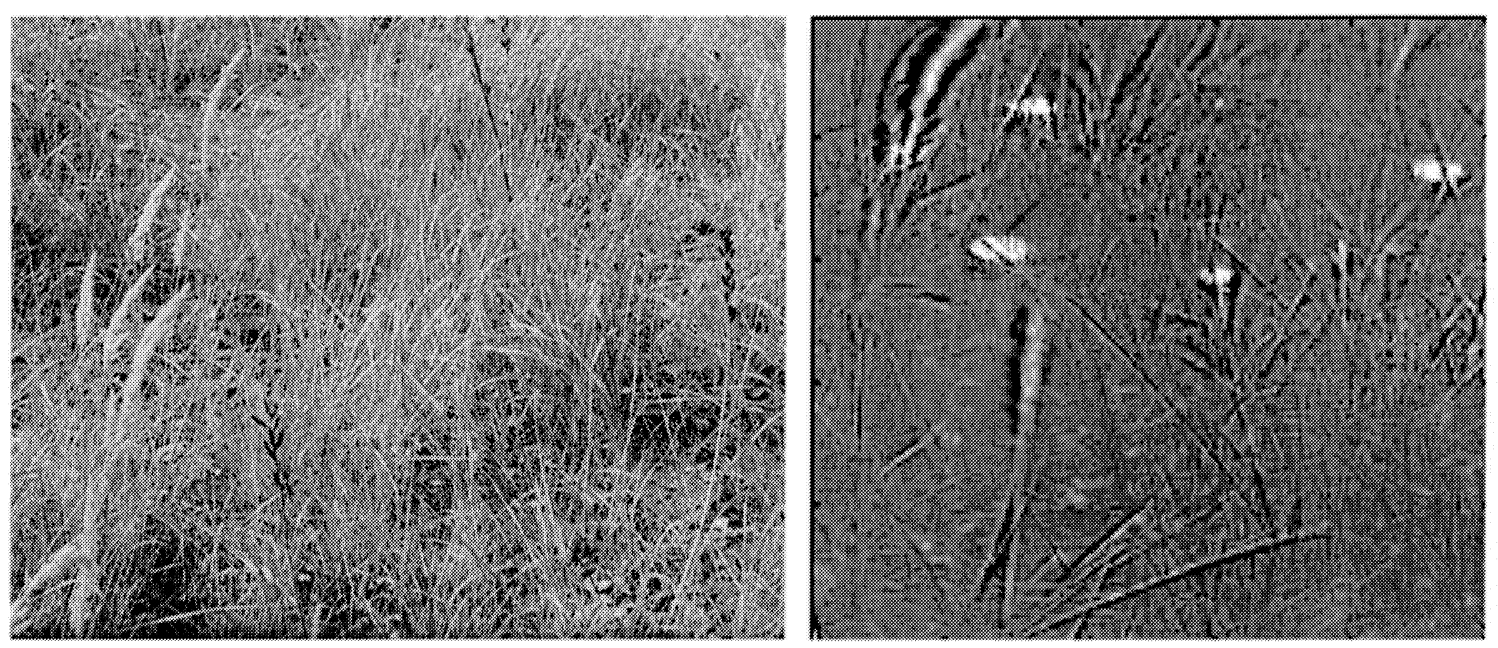

Figure 4: Infrared polarisation contrast (right) of mines partially covered by vegetation (visual image left)

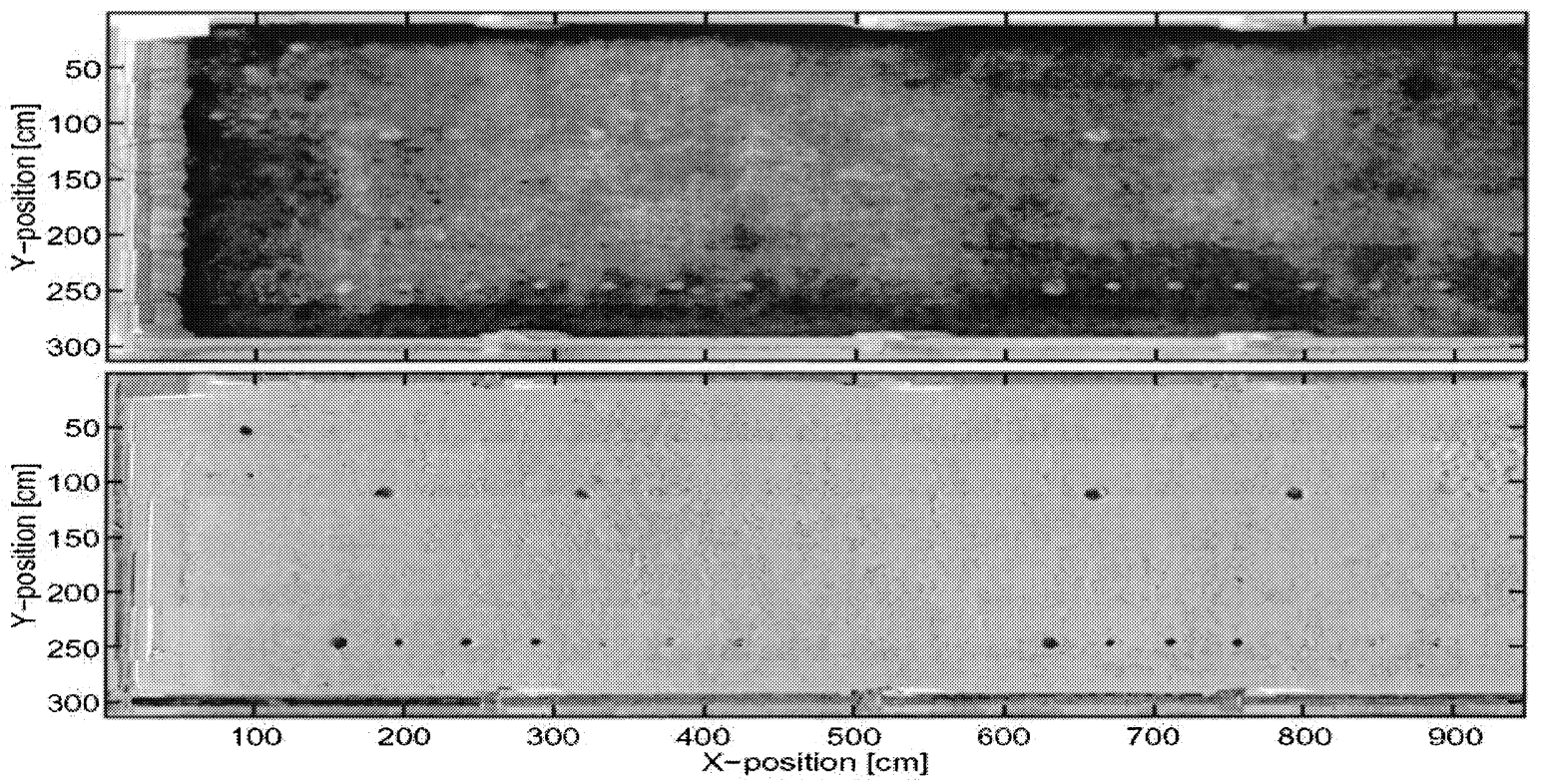

Figure 5: Polarised infrared images measured form a moving platform. Total intensity in the picture above, maximum ratio in intensity between the two perpendicular polarisation angles.

\subsection{Neutron Backscattering.}

The DUNBLAD (Delft University Neutron Backscattering Landmine Detector) project at TUD-IRI focuses on neutron backscatter detection (figure 6). The neutron backscattering technique has successfully been used to detect landmines in relatively dry sandy soils. For this purpose a novel, ergonomic detector system was constructed (figure 7). Tests show that both anti-tank (AT) and anti-personnel (AP) landmines could be detected down to at least five centimeters of depth. These measurements will be extended to more realistic environments in the near future. The use of a pulsed neutron generator will further improve the detection system. Furthermore, a neutron backscattering imager is under development. The project is presented in [7]. 


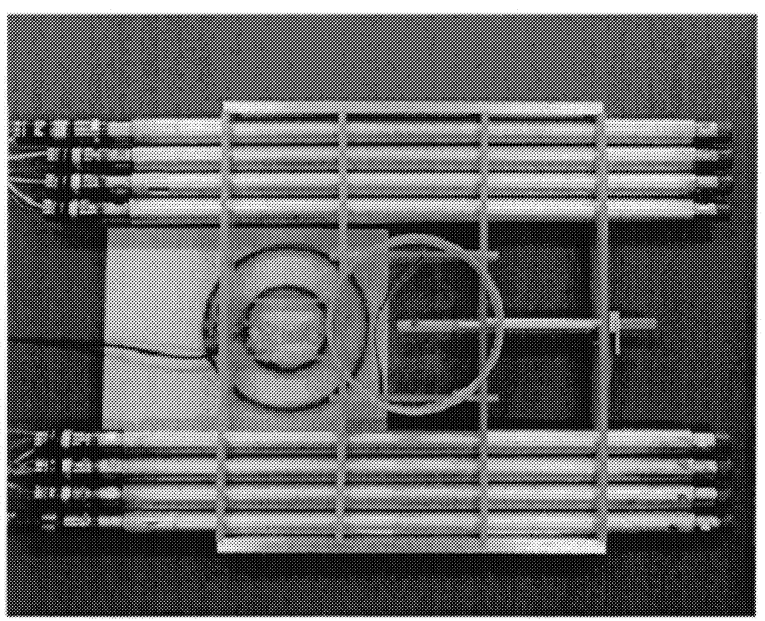

Figure 6 Photograph of the detector-head. The source, which is on the end of a $20 \mathrm{~cm}$ long stick, is placed at the center. A glass heavy water container (diameter $17 \mathrm{~cm}$, height $5 \mathrm{~cm}$ ) is positioned directly above it. To the left of it one can see the two coils of the metal detector. At both sides four $50 \mathrm{~cm}$ long helium-tubes are placed.

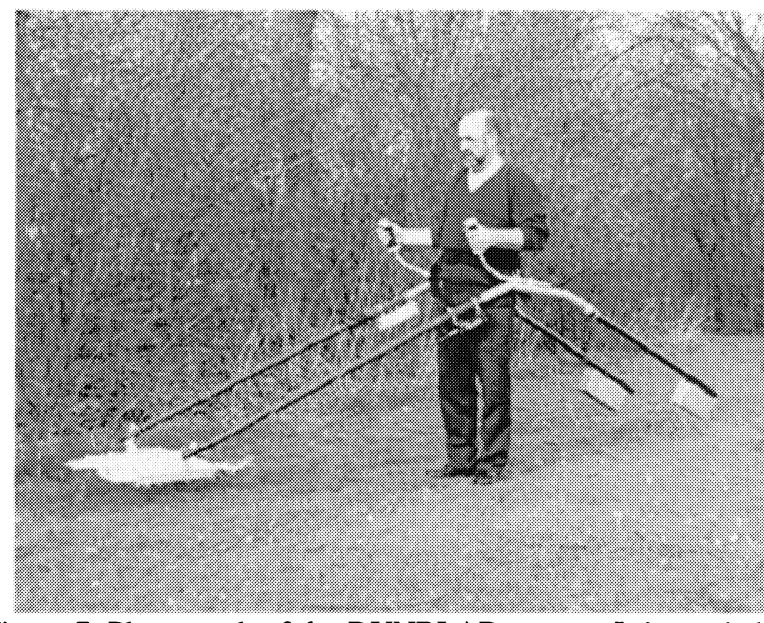

Figure 7: Photograph of the DUNBLAD system. It is carried by the deminer using a comfortable harness. The audio signals from both detectors are clearly distinguishable.

\section{SENSOR FUSION}

In the various detection scenario's, the performance of the sensors will suffer from false alarms. Combination of complementary sensors will not only increase the detection rate, but will also increase the system performance with respect to the false alarm rate.

Several sensor fusion schemes have been used in relation to landmine detection [8], [9]. In order to collect data to test the sensor fusion procedures, several multi-sensor data sets have been collected, including the data of GPR, MD and several EO sensors, looking at the same test lanes. The most recent set is the result from the joint TNO-TUD-IRCTR measuremtn campaign in Summer 2002. These data sets are available for co-operation projects, with other partners. Example of previous co-operations have lead to several joint publications [10], [11].

An important issue for sensor fusion is the registration of the data with respect to the ground position: in the sensor fusion process one has to make sure that all the sensor data are related to the correct position on the ground. For the data collection at the test facility at TNO (section 5) this is arranged by accurate positioning of the sensors. For moving platforms auxiliary sensors have to be used to monitor the movement of the individual sensors with respect to each other and to compensate for these movements. An example of an 3D-optical tracking system to enable precision data registration is given in figure 8 .

An example of a vehicle mounted multi-sensor platform is the LOTUS system. The LOTUS project combines GPR, MD and TIR detection sensors, mounted on a small vehicle. In this project, which was co-sponsored by the European Commission, TNO was responsible for TIR processing, data/sensor communication, sensor fusion, mine marking trigger and the user interface. Figure 9 shows the LOTUS vehicle with the sensors, in action in a field demonstration in Bosnia.

Figure 10 shows an example of data collected from the individual sensors, projected on the ground position and the fusion result next to the ground truth. The fusion result highlights the reduction of false alarms, compared to the individual sensors. Figure 11 gives the ROC curves of the individual sensors and the ROC curve of the fusion result for these data. Again the strong reduction is false alarms is clearly visible. Reference [12] will discuss the results of the LOTUS project in more detail. 


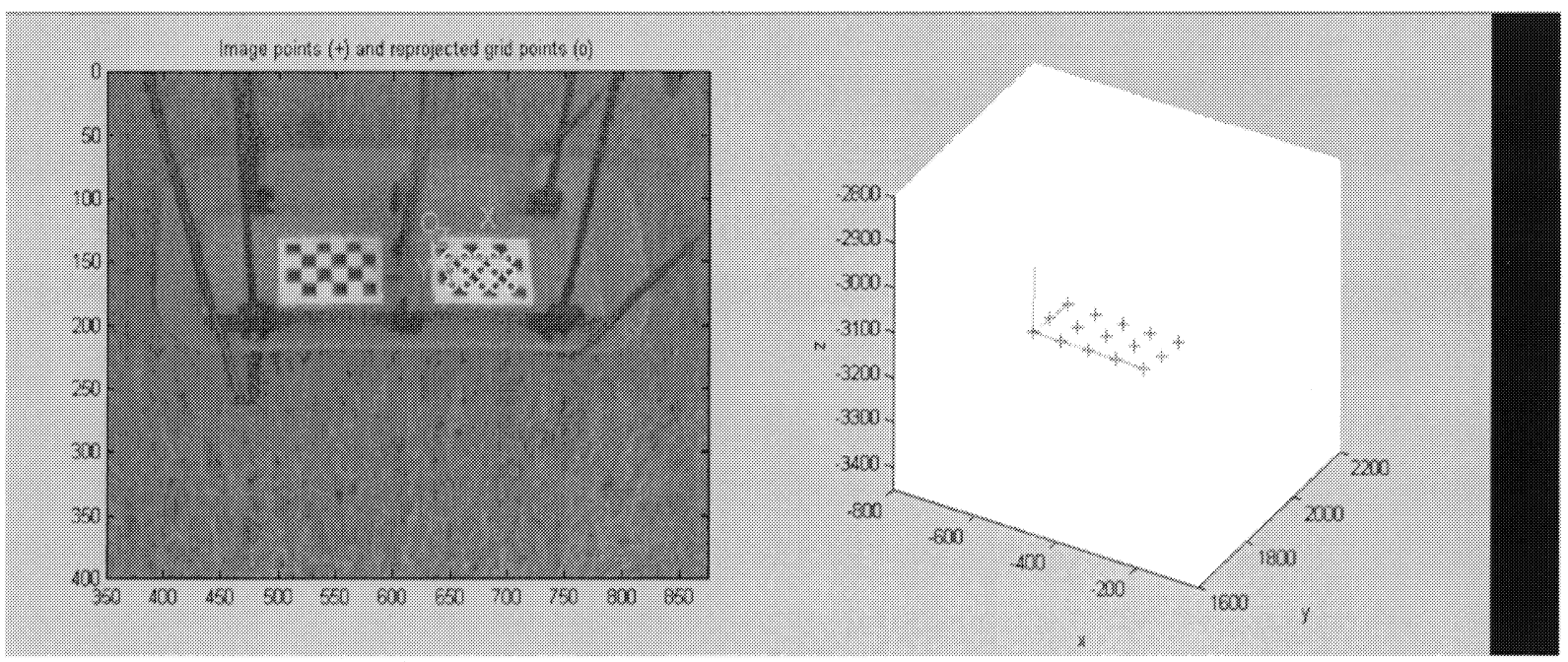

Figure 8: 3D optical tracking system, with the markers on a MD array (left) and the 3D representation of the position (right).

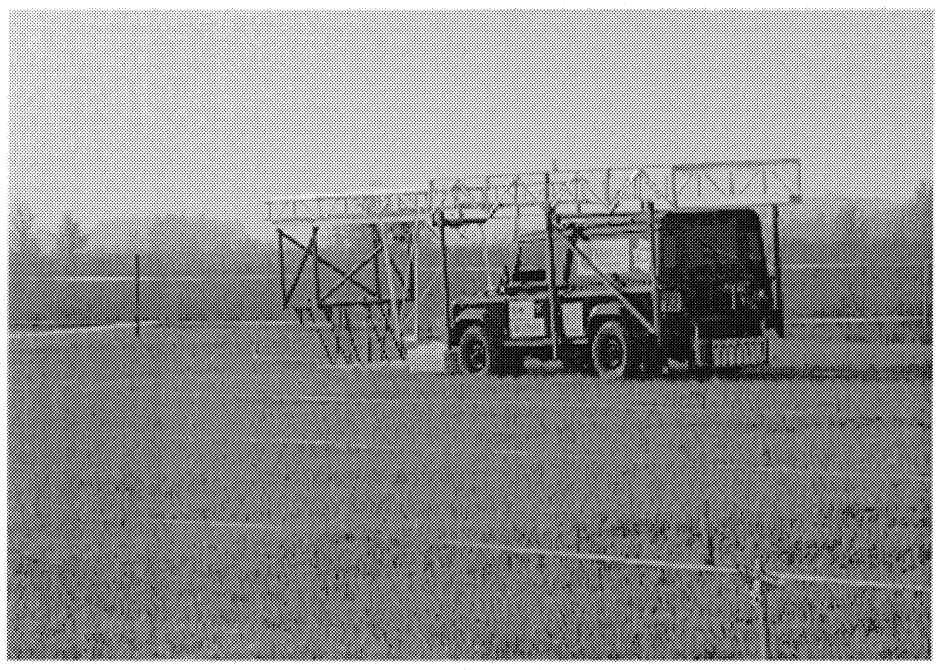

Figure 9: LOTUS Sensor platform in action in a field demonstration in Bosnia, with the MD, GPR, and TIR sensors (front) and marking unit (rear).

Apart form the data registration, another issue in sensor fusion is whether to improve the sensors to the limits of their individual detection performance or to balance their performance for an optimum combination. For example with very sensitive processing one can to extract depth information of objects from the MD signals whereas this information is more or less readily available form the GPR signals. GPR sensors need delicate processing to detect surface laid mines in the surface reflection, whereas TIR sensors more easily detect surface laid mine with the proper processing. Balancing the performance of the sensors and extracting the desired feature information form the most appropriate sensor appears to be the best solution. 

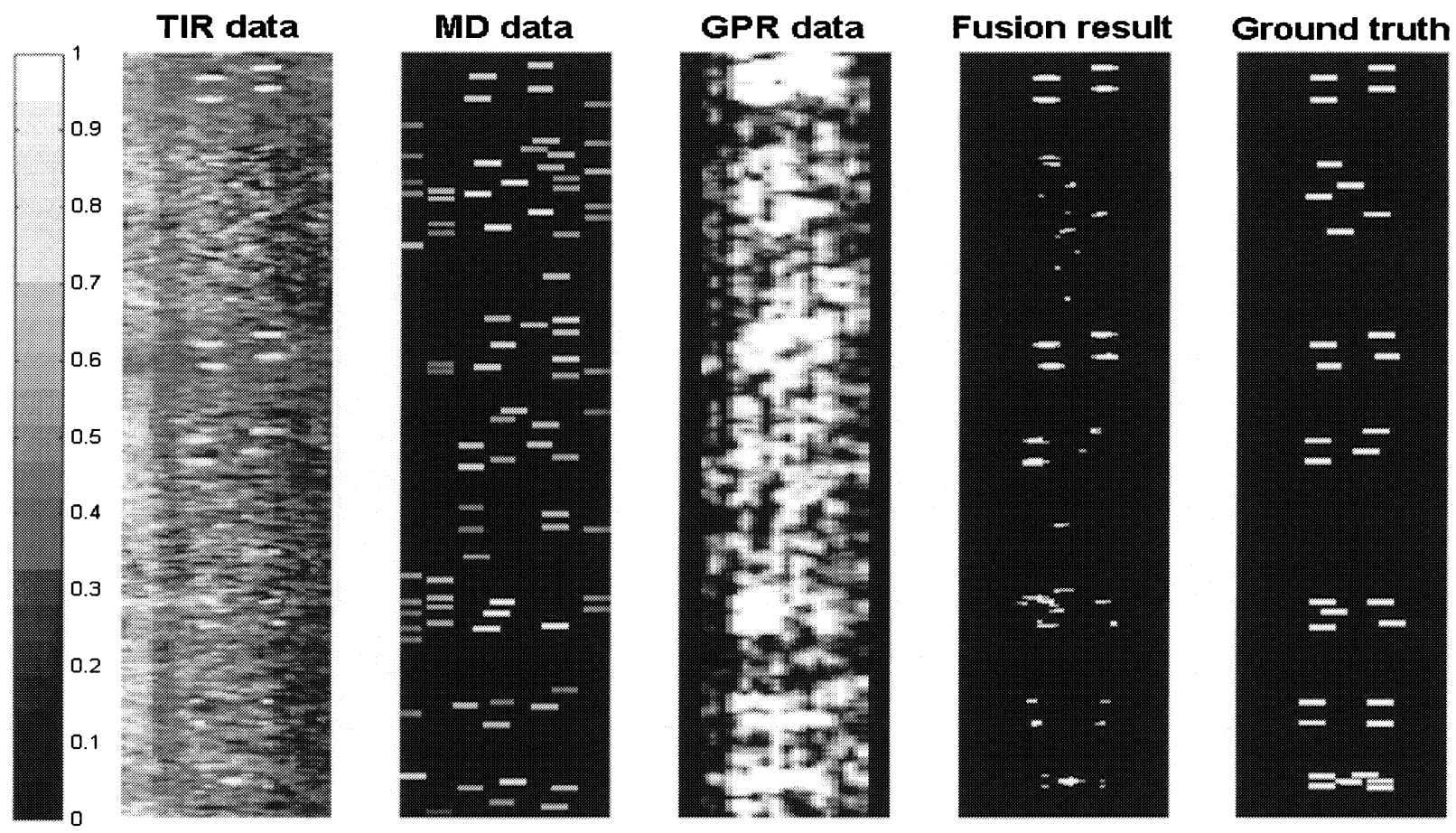

Figure 10: Example of data collected with the LOTUS TIR, MD, GPR sensors (left to right) projected on the ground position. Second from the right is the fusion result next to the ground truth (right).
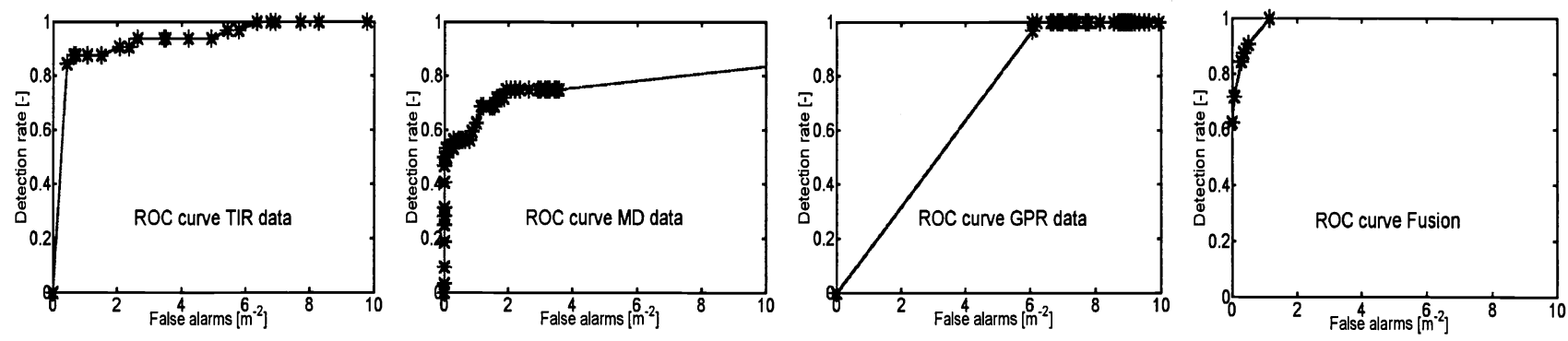

Figure 11: ROC curves of the individual sensors for the data set in figure 5 and the curve of the fusion result.

\section{MODELING THE ENVIRONMENT EFFECTS}

Tuning the sensor signal processing to the effects of the environment of the landmines, such as soil characteristics and weather conditions, on the sensor performance is an important way to improve the sensor performance, both as individual sensor as well as in a multi-sensor suite. For example the diurnal heating of the soil has a strong influence on the quality of the contribution of the TIR data to the sensor fusion process. Therefore, studies on the limitations in sensor performance due to the environment, are part of the research programme.

\subsection{Metal Detection}

It is well known that the performance of Metal Detectors is strongly affected by the susceptibility of the soil. In the IPPTC project (International Pilot Project on Technical Co-operation, with Canada, US, UK, Netherlands and EU) 25 
different commercially available metal detectors were tested on several criteria. Part of the Netherlands contribution was a test on different soils in the test facility at TNO [13].

A spin off of this project was that a working group has been formed in order to establish internationally accepted test procedures and methods, which enable to compare and interpret the results of test at different test locations. It is also understood that models are needed to extrapolate or "translate" the results from tests under controlled conditions, to the performance in specific field conditions.

\subsection{Electro-Optics}

Thermal models of the top layers of the ground are developed in order to be able to predict the infrared contrast of landmines near or at the surface as a function of the actual weather conditions and the weather history [14]. The models are validated against various data sets. Figure 12 shows a set of data of weather data for 3 August 1998. Figure 13 compares the calculated temperature with the measured thermocouple data at $5 \mathrm{~cm}$ depth.

Also for the infrared polarisation effects models have been developed [15]. Apart from the thermal behaviour of the soil and landmines, also the background clutter should be characterised as a function of the environment conditions.
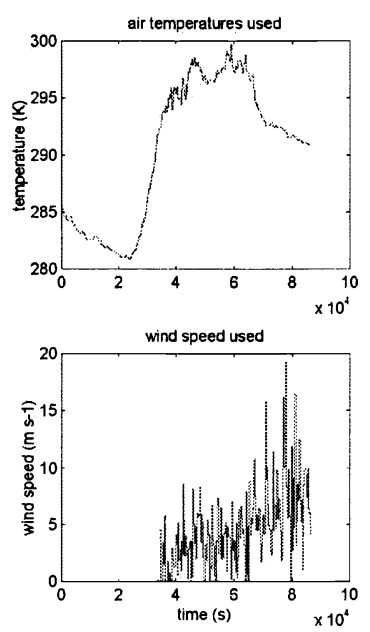

Figure 12: Weather conditions on 3 August 1998, air temperature, relative humidity, wind speed, and global irradiance (clockwise)
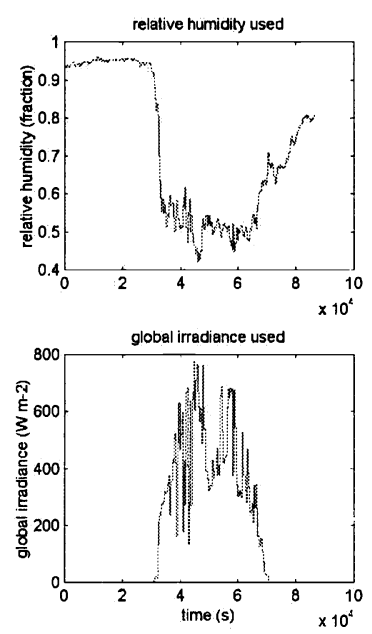

$\times 10^{4}$

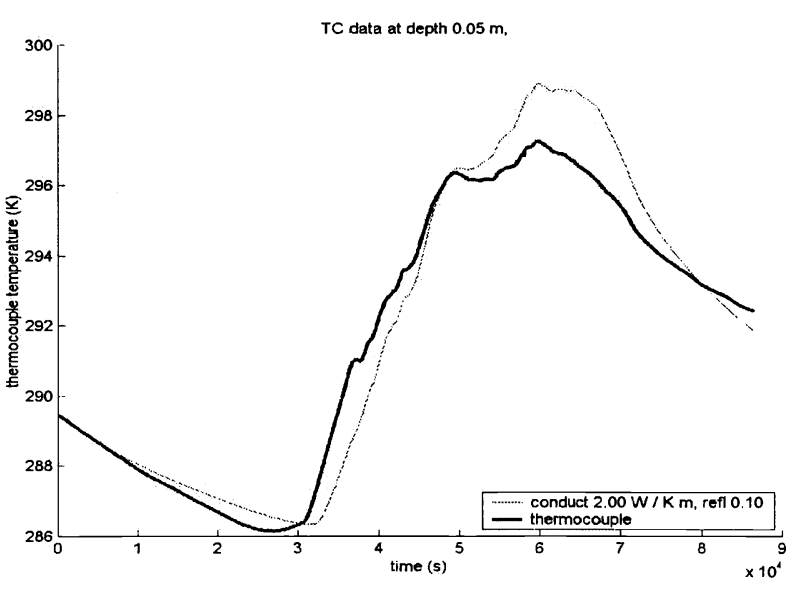

Figure 13: Comparison of the calculated temperature (red) with the measured thermocouple data (black) at $5 \mathrm{~cm}$ depth for the data in figure 12 .

\subsection{Ground Penetrating Radar}

Soil humidity is a dominant factor in GPR performance. Experiments on wetted soil are reported in [16] and [17]. In these experiments the humidity is measured with TDR probes. Figure 14 shows a typical experiment. The top part of the figure shows a vertical cross section of the soil, with three Anti Tank mines buried at $30 \mathrm{~cm}$ depth. On the left the mine is instrumented with the TDR probes (figure 15). The middle part of the figure shows a GPR-scan before wetting the soil. The two parabolas indicate the positions of the mines. The curve on the left (in blue) shows the humidity profile in the soil. The lower part of the figure shows a GPR-scan just after wetting the soil, above the left and middle mine. The soil above the mine on the right is not wetted. On the left is again the humidity profile. In the lower GPRscan the mine in the wet soil is hidden, while the mine in the dry part of the soil is still visible. Quantitative models of the effect of soil humidity are developed in co-operation with the New Mexico Tech in Socorro US.

A test of several GPR systems analogous to the IPPTC MD sensor test will provide very useful results. The lessons learned form such a test, should be a staring point for discussions on common test procedures for GPR systems. A plan for such a test with international participation from both GPR manufacturers and research institutes and universities currently in discussion. 

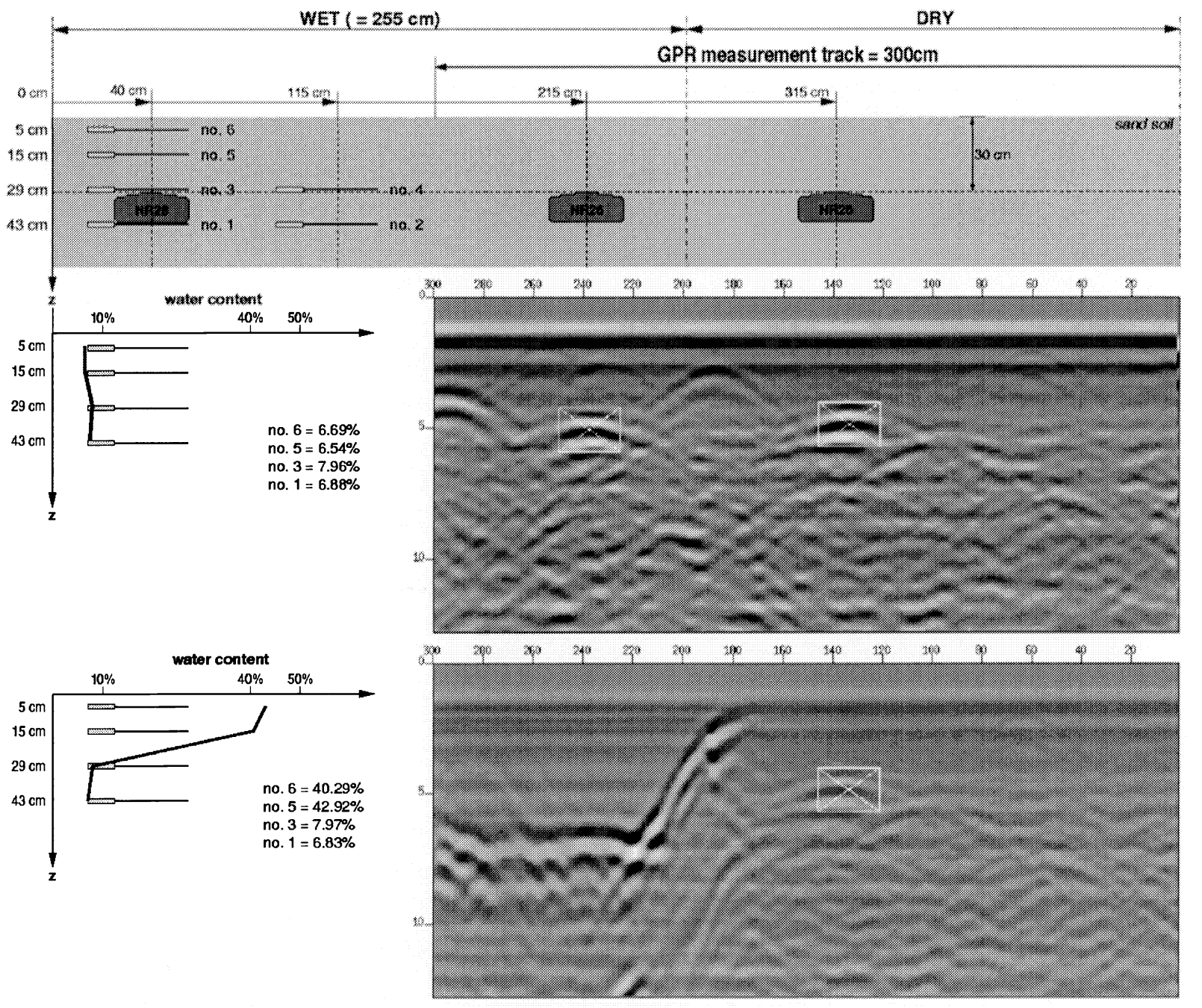

Figure 14: Typical experiment on GPR performance and soil humidity Further explanation in the text.

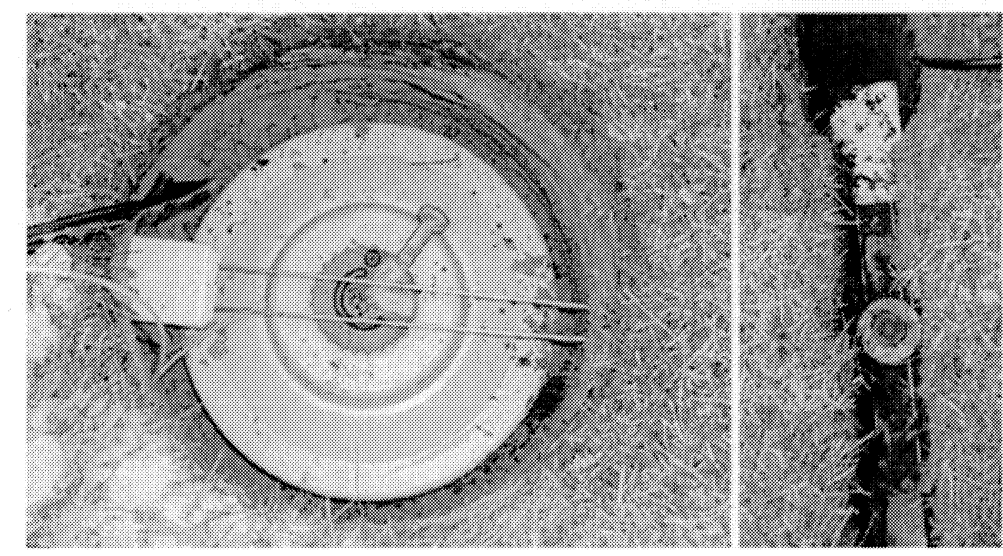

Figure 15: Examples of a TDR probes in the test lanes at TNO. 


\section{TEST FACILITIES}

In order to support the research in landmine detection, a facility has been constructed at TNO with six lanes with different soil types: sand, clay, peat, ferruginous, woodland and grassland (See figure 16). The lanes are $3 \mathrm{~m}$ wide, $1.5 \mathrm{~m}$ deep and $10 \mathrm{~m}$ long. In each of the lanes the water table is controlled individually in order to maintain a natural level for the particular soil type. TDR moisture sensing probes are available for monitoring the soil moisture during the measurements. A weather station records all the relevant meteorological data.

A metal free sensor positioning table, with an accuracy of better than $1 \mathrm{~cm}$ is controlled from a porto-cabin, where also the data recording equipment can be installed. A full description of the test facility is given in [18].

In the facility a mix of anti personnel and anti tank test mines have been buried at depths between $0 \mathrm{~cm}$ and $30 \mathrm{~cm}$. The test mines cover a range of different shapes. Both normal metal mines and very low metal content mines are represented. The characteristics of the test mines have been measured to represent the correct behaviour for MD, GPR, and TIR sensors [18]. No explosives are present in the mines. A selection of the test mines is presented in figure 17. Several of these test mines have been provided to other facilities in order to provide common reference points in data sets.

The experience with the test facilities has demonstrated the need for well-agreed and widely accepted test procedures. Well-defined performance criteria for detection capability and false alarm rates should be part of these procedures. The definition of detection capability should go beyond the percentage of detected objects, and should include information on the "degree of difficulty" for the scenario in which the mines have to be detected. For the false alarm rate one should relate the definition to the area affected by an alarm, because each alarm (true or false) needs further investigation [8].

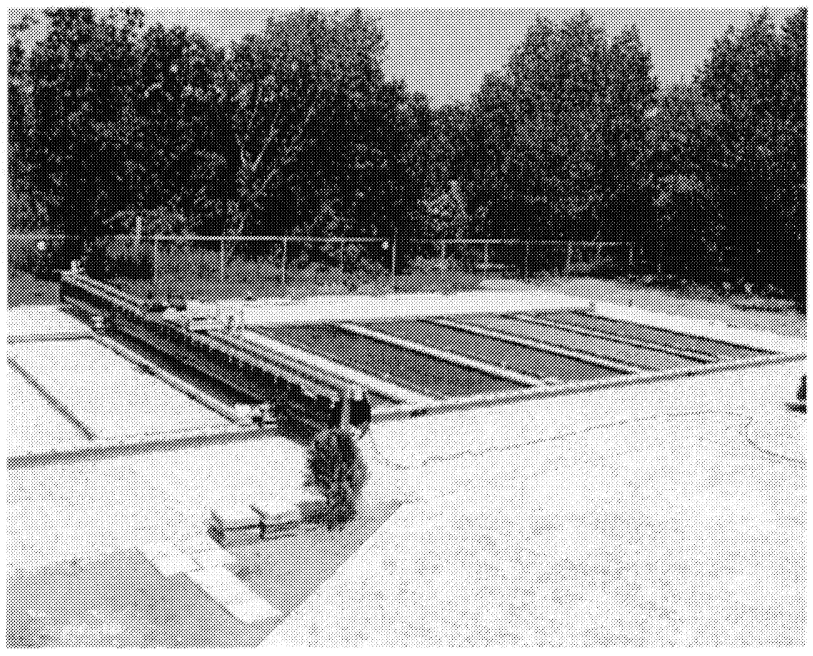

Figure 16: Test facility at TNO with six lanes with different soil types: sand, clay, peat, ferruginous, woodland and grassland (left to right).

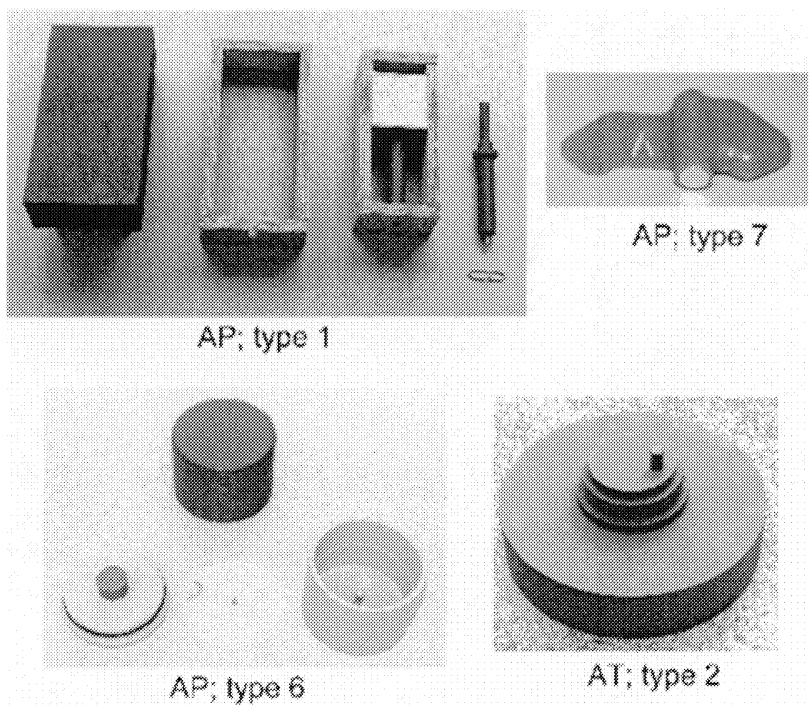

Figure 17: Selection of test mines buried at the Test facility at TNO 


\section{CONCLUSIONS}

The main conclusions from the work under the current program are summarised below:

- Multi-sensor fusion requires optimisation of sensors for extracting the desired feature information rather than optimisation of the performance of the individual sensor.

- The position registration of the data from each sensor with respect to the ground position is crucial for sensor fusion.

- The limitation of sensors as a function of weather, soil and vegetation conditions, should be taken into account when tuning the sensor fusion process.

- Proper performance models should be developed, to support this tuning in field operations.

- Considerable progress has been made in the area of GPR design.

- Well-agreed and widely accepted test procedures for all sensors should be established including performance criteria for detection capability and false alarm rates.

\section{ACKNOWLEDGEMENT}

The author would like to thank Professors Leo Ligthart and Alex Yarovoy for assistance in preparing the section on the GPR projects at TUD-IRCTR and Prof Carel van Eijk for the section on the Neutron Backscattering project at TUDIRI.

The author would like to thank Wim de Jong, Arie de Jong, Frank Cremer, Koen Benoist, Eric den Breejen, Henk Lensen, John Schavemaker, Johan van de Heuvel, Klamer Schutte, Piet Schwering, Arnold Schoolderman, Wannes van der Mark, and Jan Rhebergen for their contributions to the research programme at TNO as presented here.

The co-operation with other research institutes and universities has significantly contributed to the progress of the programme.

The financial support of the Netherlands MoD, the European Commission and STW is gratefully acknowledged.

\section{REFERENCES}

[1] M. van Mierlo, A.J. Schoolderman, TNO internal note 30916-02-01, 2002.

[2] A.G. Yarovoy, L.P. Ligthart, A. Schukin, and I. Kaploun, "Polarimetric Video Impulse Radar for Landmine Detection," Subsurface Sensing Technologies and Applications, vol.3, No.4, pp.271-293, Oct. 2002.

[3] P. van Genderen, P. Hakkaart , J. van Heijenoort, G.P. Hermans, "A Multi-requency Radar for Detecting Landmines: Design Aspects and Electrical Performance", 31st European Microwave Conference, 0-86213-148-0, pp. 249-252, London, England, 2001

[4] A.N. de Jong, H. Winkel, M.J.J. Roos, "Active multispectral Near-IR detection of small surface targets", in Detection and Remediation Technologies for Mines and Minelike Targets VI, SPIE conference 4394, 2001, pp.353-364.

[5] F.Cremer, W. de Jong, K. Schutte, Infrared polarisation and modelling applied to surface laid anti-personnel landmines" in Optical Engineering 41 (5), 2002, p. 1021-1033.

[6] J.C. van den Heuvel, V. Klein , F.J.M. van Putten, P. Lutzmann, M.Hebel, R.M. Schleijpen, "Comparison of acoustic and laser excitation in acoustic landmine detection with experimental results on buried landmines", in Detection and Remediation Technologies for Mines and Minelike Targets VIII, SPIE conference 5089-67, 2003, To be published.

[7] C. Datema, V.R. Bom, C.W.E. van Eijk, "Results of Landmine Detection with the Neutron Backscattering Method", IEEE Trans. Nucl. Sci. 48-4 (2001) p. 1087-1091.

[8] F. Cremer, K. Schutte, J.G.M. Schavemaker, E. den Breejen, "A comparison of decision-level sensor-fusion methods for anti-personnel landmine detection", Information fusion, 2(3): 187-208, Sept. 2001 
[9] P.B.W. Schwering, B. A. Baertlein, S. P. van den Broek, F. Cremer, "Evaluation methodologies for comparison of fusion algorithms in land mine detection", in Detection and Remediation Technologies for Mines and Minelike Targets VII, SPIE conference 4742, 2002, p.847-856

[10] N. Milisavljevic, S.P. van den Broek, I. Bloch, P.B.W. Schwering, H.A. Lensen, and M. Acheroy, "Comparison of Belief Functions and Voting Method for Fusion of Mine Detection Sensors", in Detection and Remediation Technologies for Mines and Minelike Targets VI, SPIE conference 4394, 2001, pp.1011-1022.

[11] P.B.W. Schwering, A Kokonozi, L.J. Carter, H.A. Lensen, E.M. Franken, "Infrared landmine detection and thermal model analysis", in Detection and Remediation Technologies for Mines and Minelike Targets VI, SPIE conference 4394, 2001, pp.219-230.

[12] J.G.M. Schavemaker, E. den Breejen, K.W. Benoist, K. Schutte, M. de Bijl, P.J. Fritz, L.H. Cohen, "LOTUS field demonstration of integrated multisensor mine-detection system in Bosnia", in Detection and Remediation Technologies for Mines and Minelike Targets VIII, SPIE conference 5089-151, 2003, To be published.

[13] Y. Das (CA), J.T. Dean (EC), D. Lewis (UK), J.H.J. Roosenboom (NL), G. Zahaczewsky (US), "International Pilot Project for Technology Co-operation", Final Report, EUR 19719 EN - 2001

[14] E.M. Franken, P.B.W. Schwering, W. de Jong, "Thermal infrared modelling for landmines, TNO report FEL-01A084, 2001.

[15] F.Cremer, W. de Jong, K. Schutte, J.T. Johnson, B.A. Beartlein, "Surface mine modeling for passive polarimetric IR" in Detection and Remediation Technologies for Mines and Minelike Targets VII, SPIE conference 4742, 2002, p.51-62.

[16] H.A. Lensen, P.B.W. Schwering, G.R. Marin, J.M.H. Hendrickx, "Measurements and modelling of soil water distribution around landmines in natural soil", Detection and Remediation Technologies for Mines and Minelike Targets VII, SPIE conference 4742, 2002, pp.417-427.

[17] J.B. Rhebergen, H.A. Lensen, P.B.W. Schwering, G.R. Marin, J.M.H. Hendrickx, "Soil moisture distribution around land mines and the effect on relative permittivity" in Detection and Remediation Technologies for Mines and Minelike Targets VII, SPIE conference 4742, 2002, p. 269-280.

[18] W. de Jong, H.A. Lensen, Y.H.L Jansen, "Sophisticated test facility to detect land mines", Detection and Remediation Technologies for Mines and Minelike Targets IV, SPIE conference 3710, 1999, p1409-1418. 